PII: S0735-1933(04)00004-1

\title{
A DEPOSITION MODEL APPLIED IN A THERMAL SPRAY PROCESS
}

\author{
J.L. Lin and H.K. Ma \\ Department of Mechanical Engineering, National Taiwan University, \\ Taipei, Taiwan, R.O.C
}

(Communicated by J.P. Hartnett and W.J. Minkowycz)

\begin{abstract}
In a thermal spray process, particles are fed into a high-velocity, high-temperature gas jet directed toward the substrate. A quantitative description of the particle impact on the substrate, as a function of particle size, injected velocity, and molten fraction, is a key to developing better models for describing the coating formation process. In this study, the results show that the positions, velocities, and temperature of the Ni particles are highly dependant on the diameter and injected velocity of the particles. The deposition-splashing boundary equation is a function of the Weber number $\left(\mathrm{W}_{\mathrm{e}}\right)$, the dimensionless maximum diameter $\left(\beta_{\max }\right)$, and the contact angle $(\Theta)$ on the substrate. The criterion of a liquid particle deposition or splashing is determined by the critical Weber number. The value of We is equal to 469.5 for $\mathrm{Re}_{\mathrm{p}}<10,000$, and 31.5 for $\operatorname{Re}_{\mathrm{p}}>10,000$. It is found that the smaller Ni particles may easily deform and then deposit or splash on the substrate, whereas particles with larger sizes $(>120 \mathrm{~m})$ do not.

(c) 2004 Elsevier Science Ltd
\end{abstract}

\section{Introduction}

Materials in powder form are molten in a plasma flame (argon or hydrogen most commonly) to form a fine spray. When the spray contacts the surface of a substrate, the fine molten droplets solidify rapidly to form a coating. Nevertheless, experimental measurements of gas and particle temperatures as well as velocities in a plasma jet are challenging due to the extremely high temperatures and velocities. Therefore, in the absence of available experimental techniques, numerical simulation has been commonly used as a method for investigating the fundamental behavior of the plasma gas and particles. Quantitative data about the thermal behavior of a particle in a plasma flame are essential to achieving uniform heating to the liquid state. Various researchers have previously modeled the thermal behavior of a single particle in plasma. For example, Fiszdon [1] accounted for non-uniform plasma conditions for a particle traveling along the centerline of the plasma reactor. Lee et al. [2] reported varying plasma and temperature jumps, and investigated the effects of relaxation time, radiation transportation, internal and temperature jumps, and investigated the effects of relaxation time, radiation transportation, internal conduction, particle shape, and 
particle charging for steady plasma. Jog and Huang [3] explored a single particle in steady plasma, and investigated the effects of gas ionization and particle charging by incorporating a model for production and recombination of electrons and ions. Wan et al. [4] simulated the actual off-centerline trajectory of the particle and the 3-D plasma gas filed by making use of the LAVA code for prediction under given power.

Mundo et al. [5] developed a theoretical approach method from the energy balance for deposition or splashing condition. For the Reynolds numbers of the particle less than 150, the solution becomes impractical and, the method is not appropriate. It is noted that a distinct correlation between the $\operatorname{Re}_{\mathrm{p}}$ and the Oh number, $\mathrm{K}=\mathrm{Oh} \mathrm{Re}_{\mathrm{p}}{ }^{1.25}$, exists only if the normal velocity component of the impinging droplets is used for these dimensionless numbers. A value of $\mathrm{K}$ bigger than 132.3 leads to incipient splashing, whereas $\mathrm{K}$ less than 57.7 leads to complete deposition of the liquid.

This study therefore aims to incorporate a deposition model into thermal spray coating. Particles with sizes ranging from $20 \mathrm{~m}$ to $120 \mathrm{~m}$ are used. The impacting positions, velocities, and temperatures on the substrate are investigated in detail. It is found that the injected velocity and size of a particle are two of the most important parameters for the spray coating process.

\section{Physical Model}

\section{Particle Spraying Modelling}

Ni particle. There is vigorous momentum and heat exchange between the injected Ni particle and the plasma gas. The basic equations describing a second-phase particle in the gas flow are as follows.

Momentum transfer. The velocity of a particle can be calculated according to the force balance on the particle

$$
F=-\frac{\pi}{8} C_{D} \rho_{g} d_{p}^{3}\left|v_{g}-v_{p}\right|\left(v_{g}-v_{p}\right)+\frac{\pi}{6} \rho_{p} d_{p}^{3} g
$$

where $v_{p}$ and $v_{g}$ are the particle and gas velocities, respectively, $p$ is the particle density, $d_{p}$ is the particle diameter, and $C_{D}$ is the drag coefficient, which was taken from Pfrender and Lee [6].

Heat transfer. Assuming that the particle is heated by convective heat transfer only, the temperature is uniform throughout the particle and there are no phase transformations in the particle, the particle temperature can be calculated from

$$
m_{p} C_{p} \frac{d T_{p}}{d t}=2 \pi d_{p} N_{u} \lambda\left(T_{g}-T_{p}\right)+m_{p} L_{m}
$$

where $m_{p}$ is the mass of the particle, $C_{\mathrm{p}}$ is the specific heat of the particle, $T_{\mathrm{p}}$ is the particle temperature, is the thermal conductivity, and $\mathrm{L}_{\mathrm{m}}$ is the latent heat. The value of $\mathrm{N}_{\mathrm{u}}$ number is the convective heat transfer coefficient, which is evaluated using the Ranz-Marshall correlation. [7]

\section{Deposition and Splashing Model}

Theoretical approach 
The phenomena of the deposition or splashing process were described as [5]. An analytic method bases on the equation of energy conservation, is described as follows

$$
E_{k}+E_{s}=E_{k}^{\prime}+E_{s}^{\prime}+E_{d}^{\prime}
$$

before impact after impact

where $E_{k}, E_{s}$, and $E_{d}$ are the kinetic, surface, and dissipated energies, respectively.

The kinetic energy before impact is defined as

$$
E_{k}=\frac{\pi \rho_{p} d_{p}^{3} w_{o}^{2}}{12}
$$

And, the surface energy before impact is described as

$$
E_{S}=\pi \sigma d_{p}^{2}
$$

The kinetic energy upon impact is decreased in deforming the droplet. In the case when a corona is not deformed and full deposition takes place, the energy becomes zero (i.e. $\mathrm{E}_{\mathrm{k}}^{\prime}=0$ ) at the maximum extension of the liquid on the surface. At the maximum diameter $d_{\max }$, the surface energy can be described as follows (Chandra \& Avedisian)

$$
E_{s}^{\prime}=\frac{\pi}{4} d_{\max }^{2} \sigma(1-\cos \Theta)
$$

where $\Theta$ is the contact angle and is defined as the tangential line at the liquid-gas interface at the point where the meniscus begins.

Compared with the kinetic and surface energies, the dissipated energy is more difficult to determine, because the velocity distribution inside the deforming droplet is unknown. According to the Chandra \& Avedisian model, a simple formula is used to determine $\mathrm{E}_{\mathrm{d}}^{\prime}$

$$
E_{d}^{\prime}=\iint_{t e V} \Phi d V d t \approx \Phi V t_{e}
$$

The dissipation per unit mass of the fluid is given by

$$
\Phi=\left(\mu \frac{\partial u}{\partial y}-\rho \overline{u^{\prime} v^{\prime}}\right) \frac{\partial u}{\partial y} \approx \rho \kappa^{2} y^{2}\left|\frac{\partial \bar{u}}{\partial \bar{y}}\right|\left(\frac{\partial \bar{u}}{\partial \bar{y}}\right)^{2} \approx \frac{\rho \kappa^{2} w_{o}^{3}}{h}
$$

where $t_{e}$ is the time for deformation and is estimated by $t_{e} \approx d_{p} / w_{o}$. The volume of the fluid (V), when it is flattened out in the shape of a disc, is defined as

$$
V \approx \frac{\pi}{4} d_{s}^{2} h
$$

where $h$ represents the height of the disc and $d_{s}$ is the splat diameter. The value of $d_{s}$ varies from 0 to $d_{\text {max. }}$. Usually, $\mathrm{d}_{\mathrm{s}} \approx d_{\max } / 2$, is recommended.

Substituting Eqs (9) and (10) into Eq (8), we can present the dissipated energy as

$$
E_{d}^{\prime}=\frac{\pi \rho w_{o}^{2} d_{p} d_{\max }^{2}}{16}
$$


Substituting Eqs (5), (6), (7), and (11) into Eq(4), and using the $\operatorname{Re}_{\mathrm{p}}$ and $\mathrm{W}_{\mathrm{e}}$ numbers with $\beta_{\max }=d_{\max } / d_{p}$, we can obtain the equation for the deposition-splashing boundary equation as follows

$$
\frac{\kappa^{2} W_{e}}{4} \beta_{\max }^{2}+(1-\cos \Theta) \beta_{\max }^{2}-\left(\frac{1}{3} W_{e}+4\right)=0
$$

The Ohnesorge number, $\mathrm{Oh}=\sqrt{\mathrm{We}} / \mathrm{Re}$, can then be represented as

$$
O h= \pm \sqrt{\frac{3(1-\cos \Theta) \beta_{\max }^{2}-12}{(1-0.75) \operatorname{Re}_{p}^{2}}}
$$

Equation (12) is the deposition-splashing boundary equation, which is defined as a function of the Weber number $\left(W_{e}\right)$, the dimensionless maximum diameter $(\max )$, and the contact angle $(\Theta)$. However, to solve $\mathrm{Eq}(13)$, one must first determine to the values of $\beta_{\max }$ and $\Theta$.

\section{Numerical Simulation}

Basic assumptions are made as follows.

(1) The plasma is local thermodynamic equilibrium.

(2) The Ar plasma gas specific heat capacity is the function of temperature.

(3) The radiation energy losses are negligible.

(4) The plasma obeys the ideal gas law; and plasma temperature and velocity are constant across the plasma gun exit.

(5) The plasma gas thermal conductivity and viscosity are taken to be:

$<\mathrm{i}>$ independent of plasma gas composition;

$<\mathrm{ii}>$ identical to those of the primary Ar; and

$<$ iii $>$ predictable by linear extrapolation from low temperature [8].

(6) Diffusion of species because of concentration gradients is negligible compared with the high gas velocities in the plasma jet.

(7) The dissociation and ionization of gases and the recombination of the dissociated atoms or ionized ions and electrons can be treated as standard thermodynamic chemical reactions. The chemical reactions describe as fellows

$$
\begin{aligned}
& \mathrm{Ar}+\mathrm{e}^{-} \Rightarrow \mathrm{Ar}^{+}+\mathrm{e}^{-} \mathrm{Ar}^{*}+\mathrm{e}^{-} \Rightarrow \mathrm{Ar}^{*}+\mathrm{e}^{-} \\
& \mathrm{Ar}^{*}+\mathrm{e}^{-} \Rightarrow \mathrm{Ar}^{+}+2 \mathrm{e}^{-} \mathrm{Ar}+\mathrm{e}^{-} \Rightarrow \mathrm{Ar}^{+}+\mathrm{e}^{-}
\end{aligned}
$$

(8) The particles are assumed to obey Newtonian heat flow conditions, i.e. the rate of heating or cooling is determined by the surface heat transfer coefficient only, and internal heat conduction is sufficiently rapid that the particles are isothermal.

In the numerical analysis, a control volume method and the modified SIMPLER algorithm [9] are used as a numerical scheme for solving the governing conservation equations of mass, 
TABLE 1

The Plasma Input Data

\begin{tabular}{c|c}
\hline Plasma gas & $\mathrm{Ar}$ \\
\hline Anode diameter(mm) & 6.0 \\
Anode length(mm) & 12.0 \\
Arc current $(\mathrm{A})$ & 500 \\
Gas flow rate(NL/min) & 50 \\
Electric power(kW) & 15.2 \\
Plasmatron efficiency & $58 \%$ \\
Initial temperature & $11,000 \mathrm{~K}$ \\
Initial velocity & $1,000 \mathrm{~m} / \mathrm{s}$ \\
Initial degree & 0.05 \\
\hline
\end{tabular}

momentum, and energy along with the equations describing the RNG mod e 1 for turbulent effects. Numerical simulations are carried out for finding the thermal plasma characteristics in an exterior jet flame region outside the torch. The plasma spray field simulated is listed in Table 1. The grids adopted were $100 \times 160$.(see Fig.1)

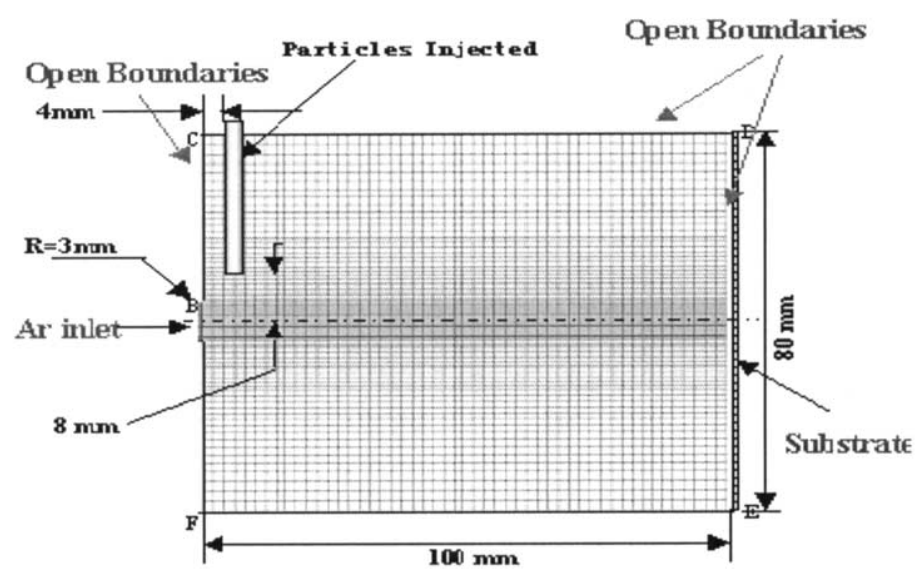

FIG.1

The calculated domain of the thermal spray.

\section{Results and Discussion}

Initially, the Ni particle was assumed to be at $\mathrm{T}_{\mathrm{pi}}=300 \mathrm{~K}$ and to be injected into the plasma jet with velocities from $1 \mathrm{~m} / \mathrm{s}$ to $32 \mathrm{~m} / \mathrm{s}$ at initial radial positions of $\mathrm{x}=4 \mathrm{~mm}$, and $\mathrm{y}=8 \mathrm{~mm}$, respectively. The flight distance was taken to be $\mathrm{L}=0.1 \mathrm{~m}$. The properties of the coating material, Ni particles, were taken from 
$\lceil 101$

\section{Effect Injected Particle Velocity}

Figure 2 shows the particle impacting the radial position of the substrate for different injected velocities. A linear relationship between particle diameter and injected particle velocities is observed. The slope decreases clearly with increasing size. When the injected velocity rises gradually, the particle will impact on the lower radial position. As can be seen, the limit of the injected velocities for the Ni particle sizes are $4 \mathrm{~m} / \mathrm{s}, 8 \mathrm{~m} / \mathrm{s}, 12 \mathrm{~m} / \mathrm{s}$, and $32 \mathrm{~m} / \mathrm{s}$, for $20 \mathrm{~m}, 50 \mathrm{~m}, 80 \mathrm{~m}$, and $120 \mathrm{~m}, \mathrm{r}$ espectively. Due to its initial momentum, the particle keeps its velocity until an increasing aerodynamic drag causes a significant deflection from the original path. The smaller particles travel along the centerline of the plasma field, thus impacting on the center of the substrate. On the contrary, particles of larger size are clearly observed along off-centerline trajectory. According to the above description, the powders are injected radially into the plasma stream, larger particles tend to pass completely through the stream, whereas smaller particles tend to reentrain the stream. Only those particles within the optimum particle size range are fully reentrain in the plasma and are deposited onto the target. Under this condition, large powders are not fully reentrain in the plasma stream and deposition efficiency is relatively low.

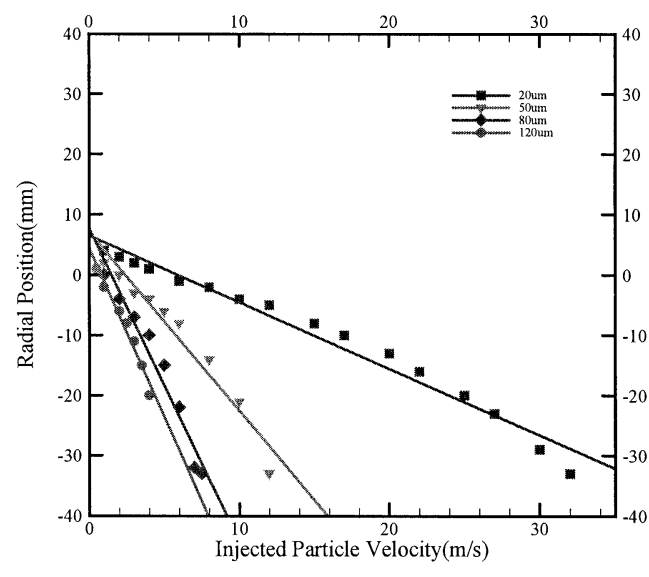

FIG.2

Relationship between radial position on the substrate and injected velocity for particles of different sizes.

The temperature and velocity profiles on the substrate are shown in Fig.3 and Fig.4, respectively. At the substrate, both the temperature and velocity increase with injected particle velocity, and the maximum temperature is attained. Then, they both decrease with the increasing injected particle velocity. In Fig.3, the maximum temperature appears at $u_{\mathrm{pi}}=10 \mathrm{~m} / \mathrm{s}, 8 \mathrm{~m} / \mathrm{s}, 5 \mathrm{~m} / \mathrm{s}$, and $3 \mathrm{~m} / \mathrm{s}$ for $\mathrm{d}_{\mathrm{p}}=20 \mathrm{~m}, 50 \mathrm{~m}, 80 \mathrm{~m}$, and 
$120 \mathrm{~m}$, respectively. At a given injected velocity, the particle temperature decreases with increasing particle size. When the temperature on the substrate is below melting point such as $120 \mathrm{~m}$ particle, the particle is remains intact. When the temperatures of the other particles $(20 \mathrm{~m}, 50 \mathrm{~m}$, and $80 \mathrm{~m})$ are equal to or above melting point, the particle is completely molten.

The velocity on the substrate is important for deposition on the surface, it is also called initial impinged velocity. Although the impinged velocity is too small to cause deposition, a high velocity will cause the droplet to splash. As seen in Fig. 4, the critical velocity is reached when $u_{p i}=11 \mathrm{~m} / \mathrm{s}, 8 \mathrm{~m} / \mathrm{s}, 3 \mathrm{~m} / \mathrm{s}$, and $2 \mathrm{~m} / \mathrm{s}$ for $\mathrm{d}_{\mathrm{p}}=20 \mathrm{~m}, 50 \mathrm{~m}, 80 \mathrm{~m}$, and $120 \mathrm{~m}$, respectively. In addition, the velocity profiles of a Ni paritcle with the injected velocities on the substrate are similar to Gauss' distribution.

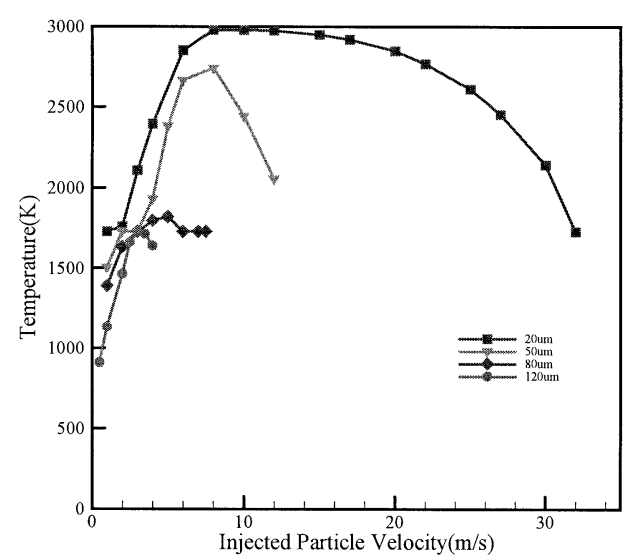

FIG.3

Relationship between particle temperature on the substrate and injected velocity for particles of different sizes.

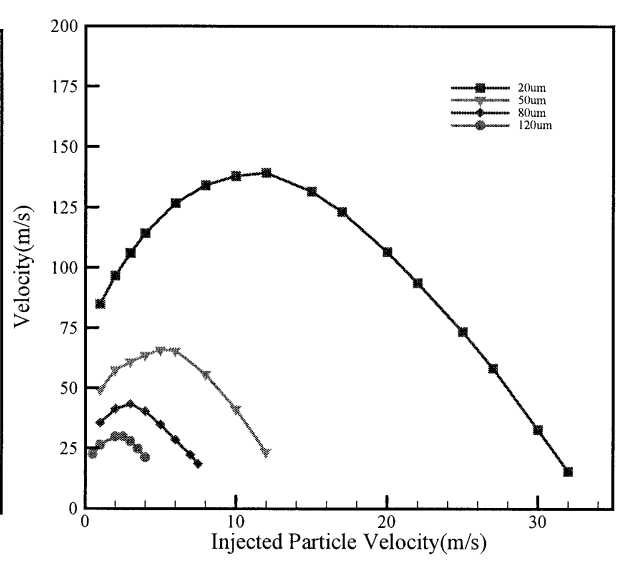

FIG.4

Relationship between particle velocity on the substrate and injected velocity for particles of different sizes.

\section{Particle Deposited or Splashed on the Substrate}

Comparisons between the data predicted by theoretical approach and the experimental data are shown in Figs.5, and 6. The model of Mundo et al. cannot use to determine the conditions of the deposition or splashing at $\mathrm{Re}_{\mathrm{p}}<100$. The prediction is in good agreement with the experimental data when using $\Theta=75^{\circ}$ and $\max =2.8$ for $\operatorname{Re}_{\mathrm{p}}<10,000$. They are also in good agreement with the measured data when using $\Theta=75^{\circ}$ and $\max =2.65$ for $\operatorname{Re}_{\mathrm{p}}>10,000$. The deposition or splashing process can be described as below with the criterion value of Weber number (We).

$$
\left\{\begin{array}{c}
W e<469.5, \text { droplets depositing } \\
W e>469.5, \text { droplets splashing }
\end{array} \text { for } \mathrm{Re}_{\mathrm{p}}<10,000\right.
$$




$$
\left\{\begin{array}{l}
W e<31.5, \text { droplets depositing } \\
W e>31.5, \text { droplets splashing }
\end{array} \text { for } \mathrm{Re}_{\mathrm{p}}>10,000\right.
$$

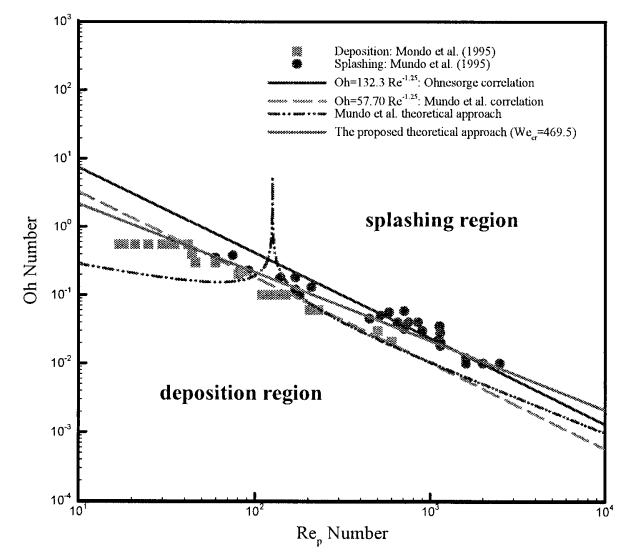

FIG.5

Limits for splashing and deposition of a droplet on the surface.

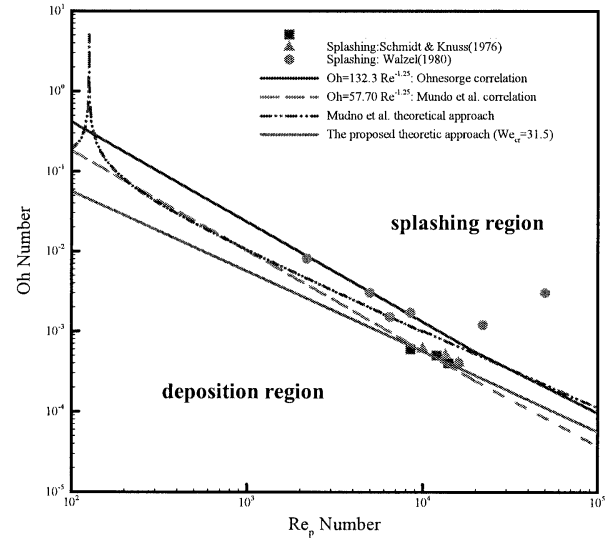

FIG.6

Limits for splashing and deposition of a droplet on the surface.

Figure 7 shows the relation of the $\mathrm{Oh}$ and $\mathrm{Re}_{\mathrm{p}}$ numbers for different particle sizes. The dash-dot line represents the Mundo model, the limits of $\mathrm{K}$ is 57.7. The long dotted line represents the Ohnesorge model, the limits of $\mathrm{K}$ is 132.3. The calculated values of $\mathrm{K}$ number are between 57.7 and 393.6. And, the ones of We number exceed than 469.5. The results show that the liquid metals impacting on the substrate have lower $\mathrm{Oh}$ and higher $\mathrm{Re}_{\mathrm{p}}$ numbers. In additional, particles of small size easily deform on the substrate, whereas the large ones $(120 \mathrm{~m})$ does not.

\section{Conculsions}

The deposition model on the substrate was developed to predict a deposition or splashing process. The following is a summary of the findings and conclusions.

(1). The deposition-splashing boundary equation can be derived as below.

$$
\frac{\kappa^{2} W_{e}}{4} \beta_{\max }^{2}+(1-\cos \Theta) \beta_{\max }^{2}-\left(\frac{1}{3} W_{e}+4\right)=0
$$

(2). The deposition process or splashing process can be described as below with respect to the criterion value of Weber number. 


$$
\begin{aligned}
& \left\{\begin{array}{c}
W e<469.5, \text { droplets depositing } \\
W e>469.5, \text { droplets splashing }
\end{array} \text { for } \mathrm{Re}_{\mathrm{p}}<10,000\right. \\
& \left\{\begin{array}{l}
W e<31.5, \text { droplets depositing } \\
W e>31.5, \text { droplets splashing }
\end{array} \text { for } \mathrm{Re}_{\mathrm{p}}>10,000\right.
\end{aligned}
$$

(3). Particles of smaller size $\left(d_{p}=20 \mathrm{~m}\right)$ travel along the centerline of the plasma field and tend to be molten and splash on the substrate, whereas the larger Ni particles $\left(d_{p}=120 \mathrm{~m}\right)$ will not melt in its flight, but rebound on the substrate.

(4). The melting Ni particles impacting on the substrate will have lower $\mathrm{Oh}$ and higher $\mathrm{Re}_{\mathrm{p}}$ numbers.

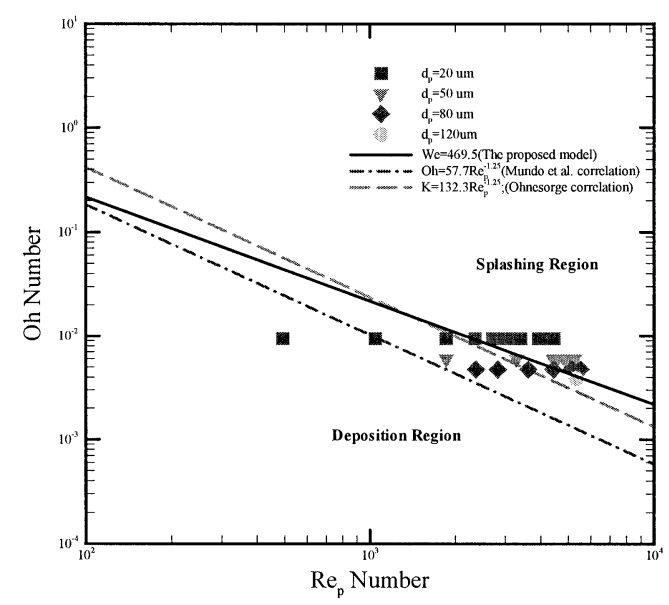

FIG.7

Relationship between $\mathrm{Oh}$ number and $\mathrm{Re}_{\mathrm{p}}$ numbers for particles of different sizes.

\section{Nomenclature}

$\begin{array}{ll}\mathrm{a}_{\mathrm{x}} & \text { spreading acceleration } \\ C_{D} & \text { drag coefficient. } \\ \mathrm{C}_{p l} & \text { liquid specific heat } \\ \mathrm{c}_{p s} & \text { solid specific heat } \\ \mathrm{d}_{\max } & \text { The maximum diameter } \\ \mathrm{d}_{\mathrm{o}} & \text { particle diameter } \\ \mathrm{d}_{\mathrm{s}} & \text { splat diameter } \\ \mathrm{G} & \text { gravitational force } \\ \mathrm{H} & \text { The height of the disc } \\ \mathrm{L}_{e} & \text { latent heat of evaporation }\end{array}$

$\mathrm{a}_{\mathrm{x}} \quad$ spreading acceleration

$C_{D} \quad$ drag coefficient.

$\mathrm{C}_{p l} \quad$ liquid specific heat

solid specific heat

$\mathrm{d}_{\max } \quad$ The maximum diameter

latent heat of evaporation 
$\mathrm{L}_{m} \quad$ latent heat of fusion

$\mathrm{Oh}$

$\operatorname{Re}_{\mathrm{p}}$

$\mathrm{T}_{\mathrm{b}}$

$\mathrm{t}_{\mathrm{e}}$

$t^{*}$

boiling temperature
Ohnecorre number II / $\mathrm{nad}$

Particle Reynolds number,

deforming time of a droplet

dimensionless deforming time constant value. $(=0.41)$

liquid thermal conductivity

solid thermal conductivity

Droplet density

Dissipation.

\section{References}

1. J .K. Fiszdon, , Int. J. Heat Mass Transfer, Vol. 22, pp. 749-761(1979).

2. Y.C. Lee, Y.P. Chyou, and E. Pfender, Plasma Chem. Plasma Process, Vol. 5, pp. 391-415(1985).

3. M.A. Jog and L. Huang, J. Heat Transfer, Vol. 118, pp. 471-477(1996).

4. Y.P. Wang, V. Prasad, G.X. Wang, and S. Sampath, Modeling of Powder Particle Heating, Melting and Evaporation in Plasma Spraying Processes,' Proceedings of the ASME Heat Transfer Division, Volume 4, pp.67-77(1998).

5. C. Mundo, M. Sommerfeld and C. Tropea, Int. J. Multiphase Flow, Vol. 21, pp.151-173(1995).

6. E. Pfender, and Y.C. Lee, Plasma Chem. Plasma Process., Vol. 5, pp. 211-237(1985).

7. W.E. Ranze and W.R. Marshall, Chem. Eng. Prog. Vol. 48, pp. 141-173(1952).

8. Y. Y.Zhao ,P.S. Grant, and B. Cantor, Modelling Simul. Mater. Sci. Eng., Vol. 8, pp. 515-540(2000).

9. S.V. Patanker, Numberical Heat Transfer and Fluid Flow, Series in Computational Methods in Mechanics and Thermal Science, McGraw-Hill, New York(1980).

10. W. Liu, G.X. Wang, and E.F. Matthys, Int. J. Heat Mass Transfer, Vol. 38, No. 8, pp.1387-1395(1995). 